\title{
La morfología en evo-devo: enfoque generativo y genealógico
}

\author{
Morphology in evo-devo: \\ generative and genealogical approach
}

\author{
ARANTZA ETXEBERRIA \\ Departamento de Lógica y Filosofía de la Ciencia \\ Universidad del País Vasco - Euskal Herriko Unibertsitatea, UPV-EHU
}

Recibido: 16-2-2013

Aprobado definitivamente: 19-2-2013

\section{RESUMEN}

La evo-devo ha sostenido, a diferencia de la biología evolutiva estándar, que la morfología puede estudiarse experimentalmente y que los procesos generativos cumplen un papel causal en la evolución. En este trabajo se examinan dos actitudes distintas dentro de la evo-devo sobre si el estudio de la morfología que sostiene el enfoque comparativo para hacer clasificaciones evolutivas puede apoyarse en una metodología causal generativa, o si debe ser estrictamente genealógico. La primera considera que el estudio de los procesos morfológicos permite hacer inferencias evolutivas más exactas, mientras que la segunda aconseja cautela al respecto. Así que pueden distinguirse dos posiciones diferentes sobre la integración de disciplinas biológicas.

PALABRAS CLAVE

CLASIFICACIÓN, DESCRIPCIÓN/EXPLICACIÓN, HOMOLOGÍA, INTEGRACIÓN, PATRONES Y PROCESOS 


\begin{abstract}
Evo-devo has maintained, unlike standard evolutionary biology, that morphology can be studied experimentally and generative processes play a causal role in evolution. In this paper two different approaches within evo-devo are examined on whether the study of morphology at the basis of the comparative approach for systematic classifications can be based in a causal generative methodology, or it must be strictly genealogical. The first one considers that the study of morphological processes enables more accurate evolutionary inferences, whereas the second is cautious about this. Accordingly, two different attitudes towards the integration of biological disciplines can be distinguished on this issue.
\end{abstract}

KEYWORDS

CLASSIFICATION, DESCRIPTION/EXPLANATION, HOMOLOGY, INTEGRATION, PATTERNS AND PROCESSES

\title{
INTRODUCCIÓN
}

UNA DE LAS SEÑAS QUE Distinguen a La BIOLOGía EVOlutiva del desarrollo (evo-devo) de la biología evolutiva estándar ha sido su preocupación por las formas o estructuras orgánicas, a las que tiende a considerar como previas a las funciones que desempeñan, y en general como punto de partida de las investigaciones biológicas. ${ }^{1}$ Para este campo de investigación la explicación causal o mecanicista de cómo se generan las morfologías en los procesos ontogenéticos es un ingrediente que debe completar la teoría de la evolución heredada de la síntesis moderna (SM). En contraste, la biología evolutiva más estándar otorga un papel meramente descriptivo a la morfología, siendo la clasificación de las formas orgánicas una labor genealógica en la que no entran en juego consideraciones sobre el origen generativo de las mismas. Aun así, el papel de la morfología en la teoría de la evolución sigue siendo un aspecto controvertido incluso dentro de la evo-devo, pues los esfuerzos por defender su carácter explicativo chocan con la enorme complejidad y diversidad de las formas vivientes, que aconseja a veces cautela a la hora de hacer inferencias desde los mecanismos de desarrollo a los procesos evolutivos (Wagner et al. 2000). A pesar del entusiasmo de autores como Pere Alberch o Simon Conway Morris, entre otros, en defensa de una biología predictiva, este asunto es aun controvertido y este trabajo está dedicado a este problema.

Uno de los pilares de la Síntesis Moderna (SM) ha sido la distinción de Mayr (1961) entre las causas próximas y las causas últimas de los fenómenos biológicos. Las causas próximas son influencias mecánicas, inmediatas de los rasgos en la operación e interacción de elementos estructurales. Las causas

1 Nuño de la Rosa (2011) examina en detalle el concepto de forma en la biología contemporánea. 
evolutivas ${ }^{2}$ explican por qué los organismos tienen un rasgo determinado en lugar de otro (Laland et al. 2011). Aunque los dos tipos de causas no son alternativas y responden a diferentes cuestiones, la biología evolutiva estándar ha estado fundamentalmente basada en las causas o explicaciones evolutivas. Para Mayr, el énfasis en las causas mecánicas que sucede al cartesianismo desemboca en el vitalismo, mientras que la biología evolutiva permite una salida a aquella amenaza comprendiendo que las causas orgánicas son evolutivas, y no pueden entenderse sólo en términos mecánicos. Posiblemente uno de los apoyos fundamentales de la evo-devo haya sido la pérdida de confianza en esa noción de genes como portadores de información que se ha generalizado en la biología del cambio de siglo, pues la distinción de Mayr descansa en su forma de entender la información genética como un elemento causal próximo que está configurado evolutivamente.

La SM señala que para dar cuenta de cualquier hecho biológico actual, explicar el funcionamiento de un órgano, o la forma de una estructura orgánica, además de hacer explícitas sus causas próximas, hay que recurrir a la historia evolutiva de la que todo fenómeno biológico actual es producto. Es por ello que la teoría evolutiva se presenta como la «teoría del todo» en biología. Sin embargo, para explicar la evolución, o un fenómeno evolutivo, que ocurre a lo largo de un amplio espacio temporal, no se hace uso generalmente de explicaciones basadas en causas próximas, procesos «aquí y ahora», ya que las poblaciones de organismos actuales disponibles para la investigación son el producto final de ese proceso, y se piensa que no pueden servir para explicar qué sucedió en el pasado. De este modo, la comprensión de la organización actual de un fenómeno biológico depende de su historia evolutiva, mientras que la comprensión de la evolución queda fuera del dominio de investigación de las causas próximas, y por lo tanto es independiente de la organización.

La consideración de que las causas próximas puedan ser valiosas más allá del contexto de la biología funcional fisiológica y que vayan a permitir elucidar aspectos nuevos de los procesos evolutivos es probablemente la principal promesa que inaugura la evo-devo. En efecto, la comprensión de los mecanismos de la evolución fenotípica, un mero epifenómeno para la concepción estándar basada en los genes, sería el principal desafío de la evo-devo. En consecuencia, la evo-devo pone en cuestión la distinción de Mayr. El renovado interés por el

2 En algunos de sus trabajos y adoptando un enfoque informacional para entender el genoma, Mayr identifica las causas próximas con el proceso de decodificación de un programa genético, el contenido del cual es el resultado de causas últimas. La evolución, por tanto, de ocuparía de las causas últimas que dan origen a nuevos programas genéticos o a la modificación de los mismos: «Son los acontecimientos del pasado que alteraron el genotipo. No se pueden investigar con los métodos de la física y de la química, sino que hay que reconstruirlos mediante inferencias históricas» (Mayr 1998, p.86). 
desarrollo, después de la reconocida exclusión de la embriología de la síntesis (Hamburger 1980), sugiere que la relevancia de factores causales próximos en las explicaciones evolutivas es más importante de lo que podría haber parecido. Uno de los principales mensajes que nos transmite la evo-devo es que para entender el cambio evolutivo hay que poder entender el cambio en los sistemas de desarrollo, para saber por qué existe cierta característica es necesario estudiar, además de su historia selectiva, no sólo cómo se construye, sino también cómo evolucionan los propios procesos de construcción. Estudiando las propiedades físicas de los materiales más básicos de los sistemas de desarrollo (Newman \& Comper 1990) se pueden extraer también propiedades genéricas de los mismos, propiedades que determinan el desenlace del proceso tanto «aquí y ahora» como en el pasado.

El enfoque generativo es importante tanto en los estudios del origen de la vida como en otros orígenes en la evolución, ligadas a la morfogénesis multicelular. Newman (2011, p. 338) compara ambas situaciones y considera que aunque es bastante complicado estudiar los procesos ligados al origen de la vida en términos de sistemas dinámicos que sigan un conjunto de principios dinámicos simples, es razonable estudiar los principios que rigen la morfogénesis de los multicelulares en las propiedades de sus materiales, pues su evolución se remonta hasta el Cámbrico y no hasta el origen de la vida. Así, comparando procesos de desarrollo de taxones relacionados evolutivamente se pueden encontrar indicios de qué posibles rutas evolutivas están disponibles.

Los últimos descubrimientos sobre el desarrollo han puesto de manifiesto que los procesos de desarrollo actuales son muy conservadores, es decir, que los mecanismos de desarrollo de los organismos actuales han sido usados repetidamente en la evolución. El estudio de estos mecanismos de desarrollo, de sus causas próximas, puede por lo tanto ayudar a explicar fenómenos evolutivos originados en el pasado. Esto no implica que sea posible sustituir un tipo de explicación por otra, la evolución es un proceso tremendamente oportunista (Jacob 1977), en el que siempre se produce una selección entre alternativas, sino que el estudio de los principios fundamentales de la organización biológica va a servir para acotar el enorme rango de historias posibles que permite generar la explicación histórico-selectiva.

En este trabajo se consideran estas dos actitudes diferentes con respecto a cómo se debe proceder científicamente en el estudio de las morfologías orgánicas, considerando si la morfología puede basarse en la búsqueda de causas que expliquen los patrones observados. Las secciones II, III y IV presentan dos diferentes formas de entender esta cuestión desde el punto de vista del método comparativo. Las últimas secciones ofrecen una discusión y conclusiones. 


\section{SOBRE EL CARÁCTER FORMAL VERSUS EXPERIMENTAL DE LA MORFOLOGÍA}

En general en la Biología Evolutiva la noción de patrón o forma se refiere a las filogenias y no a las morfologías: los patrones reflejan el orden genealógico de la naturaleza. Así, se distinguen habitualmente los campos descriptivos, dedicados a los patrones evolutivos (sistemática, paleontología, anatomía comparada) y los explicativos, que deben dar cuenta de los procesos causales de la evolución (deriva, selección). Por tanto, cuando en la biología evolutiva se considera el papel de la morfología, algunos autores proponen que tiene un rol puramente formal o descriptivo. Así, Ghiselin escribe: «[M]orphology is a formal, descriptive science, one that has to be conjoined with other disciplines if it is to tell us anything about causes. One might say that it provides the data that have to be explained in terms of something else» (2006, p. 310).

Sin embargo, en la Biología Evolutiva del Desarrollo (o evo-devo) estas nociones cambian, pues en esta área se subraya la relevancia de la morfología y los patrones orgánicos en el estudio de la evolución. El concepto de formación de patrones (pattern formation) tomado de la física de los sistemas dinámicos imbuye la idea de los procesos morfogenéticos como procesos dinámicos en los que las dinámicas complejas de los componentes desembocan en la formación de los patrones característicos de los organismos y sus partes.

Aun así, en esta perspectiva hay diferentes enfoques para estudiar los patrones y los procesos y la cuestión que permanece abierta es si la morfología podría basarse en la búsqueda de causas para explicar los patrones, o si la variedad de los mismos es tal que no pueden ordenarse en base a los mecanismos subyacentes. Hay una tradición, con autores como Wake (1982) o Alberch (1985), que ha defendido que la forma y la morfología cumplen un papel causal funcional, que puede estudiarse experimentalmente. Así, Wake dice: «Today we see [...] a renaissance in morphology. [...] While morphologists have long relied on the comparative method, and in fact the comparative method may be anatomy's outstanding contribution to science in general, the new morphology incorporates as well the experimental method. I define functional and evolutionary morphology as that field of science which examines the evolution of form by combining comparative and experimental methods of analysis» (1982, p. 605).

En efecto, para la biología evolutiva estándar la morfología es una disciplina descriptiva y la explicación de las estructuras es evolutiva (genética de poblaciones). Desde esta perspectiva los patrones reflejan el orden genealógico o filogenético de la naturaleza, orden que se atribuye a los procesos causales evolutivos. En cambio, la biología del desarrollo en la que se basa la evo-devo, busca una explicación causal, mecanicista de la morfología y su generación en el desarrollo ontogenético. Así la evo-devo subraya la relevancia de la morfología 
así entendida y los patrones morfológicos asociados a sus formas generativas para el estudio de la evolución.

En otro trabajo (Nuño de la Rosa y Etxeberria 2011) examinábamos esta cuestión en base a las diferentes propuestas que ha habido en la biología del desarrollo sobre el uso y la conceptualización de patrones (o estructuras) y procesos (o mecanismos causales). ${ }^{3}$ Ahí se distinguen tres enfoques fundamentales en la historia de la evolución y el desarrollo. El primero es básicamente recapitulacionista y trata de hacer inferencias evolutivas en base a los paralelismos y los cambios observados entre los dos procesos (tales como las heterocronías). El segundo es decididamente experimentalista y considera que lo que se averigüe sobre los mecanismos causales que tienen lugar en el desarrollo debe servir para estudiar la evolución de forma causal y hasta cierto punto predictiva. Finalmente, el tercero sugiere cautela ante lo que se percibe como un peligro del segundo enfoque, puesto que la disociación entre los procesos ontogenéticos y los patrones resultantes que se observa en la evolución obliga a considerar que tanto procesos como patrones deben explicarse en sí mismos por separado, sin que los procesos puedan asociarse causalmente a determinados patrones resultantes ni viceversa. Una consecuencia de esta llamada a la prudencia podría ser el retorno a un enfoque comparativo de corte nuevamente descriptivo o formal, basado en el método genealógico, aunque también hay propuestas de fundamentar el enfoque comparativo en un marco global de carácter generativo.

En consecuencia, dentro de la evo-devo hay un cierto desacuerdo sobre los méritos respectivos del enfoque experimental o el formal para estudiar la morfología, que tiene consecuencias para la forma de entender el papel que el estudio del desarrollo tiene a la hora de establecer clasificaciones evolutivas.

\section{El ENFOQUe COMPARATIVO DE CARÁCTER GENERATIVO}

El enfoque comparativo produce clasificaciones de la diversidad en base a juicios sobre semejanzas y diferencias. Un concepto clave es el de homología, que se refiere a la presencia de la misma parte corporal u otro rasgo orgánico en diferentes tipos de organismos. Darwin redefinió este concepto pre-evolutivo ${ }^{4}$ para atribuir la mismidad a un ancestro común, de forma que las homologías permitan el establecimiento de genealogías (Etxeberria y Nuño de la Rosa 2009). Una de las grandes aspiraciones de la evo-devo generativa ha sido el de devolver el anclaje morfogenético causal a las clasificaciones sistemáticas de la

3 El uso de los conceptos de patrón y proceso en la biología evolutiva es bien distinto, pues allí patrón se refiere al esquema genealógico resultante del proceso evolutivo entendido en términos de la genética de poblaciones (Nuño de la Rosa y Etxeberria 2011).

4 Recientemente se dispone de una nueva edición del trabajo clásico de Owen en castellano (Owen 2012). 
cladística, que se perciben como despreocupadas de la base mecanicista común de la diversidad morfológica y dirigidas a meramente salvar los fenómenos.

Así, muchos autores han reclamado que lo que la cladística llama sinapomorfías $^{5}$ no se corresponde con las homologías entendidas desde una base ontogenética y han destacado la presencia de dos grandes concepciones de la homología en la biología evolutiva actual (Wagner 1989, Abouheif 1997, Brigandt 2002, Amundson 2001). El concepto filogenético de homología considera que dos caracteres son homólogos cuando se derivan de un rasgo equivalente en el ancestro común. El objetivo es distinguir las homologías de las analogías, entendidas tradicionalmente como convergencias adaptativas producidas por la selección natural ante parecidas demandas ambientales. Esta definición de homología encaja con los objetivos de la sistemática dedicada a identificar sinapomorfías, es decir, semejanzas orgánicas aparentes que, a través de su congruencia con datos sobre otros caracteres, permiten rastrear filogenias para, en último término, ordenar el mundo viviente mediante árboles filogenéticos verosímiles (Brigandt 2002). Esta línea de investigación no afirma nada sobre la naturaleza de las partes estudiadas, mientras que el interés de la evo-devo por los caracteres homólogos no es meramente clasificatorio. Asumiendo la crítica de Gould y Lewontin (1979) al atomismo del adaptacionismo, el concepto biológico de homología se propone como objetivo la búsqueda de formas «naturales» de reconocer las partes orgánicas, y la explicación causal (ontogenética) de las mismas. Considera que las «estructuras de dos individuos son homólogas si comparten un conjunto de constricciones de desarrollo, causados por mecanismos auto-regulativos de diferenciación orgánica. Estas estructuras son partes del fenotipo individualizadas en el desarrollo» (Wagner 1989). Las partes o caracteres de los organismos se consideran individualizados por su forma o estructura (o proceso), regenerada en cada individuo mediante el desarrollo. De este modo, las semejanzas morfológicas pueden comprenderse en términos de procesos generativos compartidos, y la variedad, en términos de sus transformaciones.

El trabajo de David Wake sobre salamandras proporciona un ejemplo de enfoque integrador de diferentes tipos de investigación (funcional, causalmecanicista, histórica) en un gran proyecto centrado en la investigación de un sólo taxón (Wake 2009). El objetivo del mismo es relacionar los principales patrones observados en las múltiples especies del taxón con los procesos de desarrollo que subyacen a su construcción. Ello permite subrayar los aspectos más causales basados en constricciones de desarrollo sin desestimar la influencia de los procesos adaptativos. Sus argumentos a favor de una biología integradora

5 Un carácter derivado que define un grupo monofilético de organismos; permite reconstruir las filogenias de forma genealógica. 
son la propia integración de los taxones biológicos que muestran una unidad de tipo que se puede estudiar teórica y experimentalmente. Otros miembros del equipo de Wake, como Pere Alberch, subrayaron este aspecto integrador basado en un marco teórico formal que permite estudiar las variedades como temas (Alberch 1989).

Por otro lado, desde este marco también se suscitan cuestiones sobre la distinción clásica entre homología y analogía. Wake (1991) ha preferido hablar de homoplasia en lugar de analogía, para indicar que la semejanza de los rasgos que no se atribuyen a un ancestro común detectado podrían no ser resultado de la selección natural, sino de convergencia, paralelismo, o reversiones basadas en que todos ellos comparten el desarrollo en algún grado (Wake et al. 2011, p. 1032). Como consecuencia de este enfoque generativo es que las distinciones dicotómicas entre homología y paralelismo, o entre paralelismo y convergencia tienden a diluirse en principio, de forma que el juicio es dependiente del nivel jerárquico en el que se plantee una investigación y de la naturaleza de la misma. Abouheif (2008, p. 4) propone que el paralelismo podría ser una transición entre caracteres verdaderamente homólogos y verdaderamente convergentes, y que las dificultades deben plantearse distinguiendo el nivel en el que se analizan los rasgos.

\section{EL ENFOQUE COMPARATIVO DESCRIPTIVO O GENEALÓGICO}

El énfasis en la importancia de las causas próximas y de los procesos ontogenéticos ha sido uno de los motivos principales/centrales de la evo-devo más dedicada a subrayar los aspectos «devo» o de desarrollo de este campo de investigación. Por su parte, la cara evolutiva (o «evo») se asienta, entre otros factores, en la defensa del método comparativo y la sospecha de las inferencias realizadas en base a los organismos modelo. Así, Minelli (2003) se queja de que el encuentro entre la biología del desarrollo y la biología evolutiva aún no se ha producido, pues ambas continúan ancladas en sus respectivas «metafísicas». En su parecer la primera sigue siendo «finalista», y presta más atención a las estructuras adultas de los organismos que al proceso complejo de la ontogenia del organismo, mientras que el enfoque basado en el progreso hace tiempo que ha sido abandonado en la teoría de la evolución (p. xvi). Su propuesta de acercamiento requeriría prestar más atención al enfoque comparativo que permitiría trascender la información obtenida del estudio experimental de los organismos modelo para integrar aspectos descriptivos típicos del enfoque comparativo. Así, el desarrollo adquiere un sentido propio, no sólo como proceso para obtener un organismo adulto, sino un elenco variado de patrones cada uno de los cuales debe ser estudiado en sí mismo.

A partir de estas ideas Scholtz $(2008,2010)$ defiende un enfoque comparativo de carácter genealógico frente al experimental. Su argumento es que los 
mecanismos de desarrollo no son mecanismos evolutivos, porque la evolución puede alterar profundamente los procesos de desarrollo: «attempts to explain homology based on (mechanisms of) development creating a distinct pattern are flawed because there is ample and increasing evidence that development is free to evolve independent of the resulting homologous structure at all levels starting from genes, via cell division, to morphogenesis and organogenesis». (Scholtz 2010, p. 57). De hecho, la variabilidad de las rutas generativas de patrones sugiere que no siempre se correlacionan patrones homólogos con procesos generativos. En consecuencia la independencia evolutiva entre patrones y procesos implica que no hay una relación causal necesaria entre estadios ontogenéticos que permita hacer inferencias. En la evolución los procesos de desarrollo pueden considerarse en sí mismos, sin tener en cuenta los patrones a los que dan lugar, mientras que en el desarrollo aparece una gran diversidad de formas, la morfología adulta no es el resultado del desarrollo sino un estadio entre muchos. Por ello defiende un método analítico para examinar formas en términos de estructuras o patrones, de forma que el papel de la morfología sea la comparación analítica de todas las estructuras morfológicas que aparecen en el desarrollo y la evolución.

\section{Discusión: ¿DOS MODELOS DE INTEGRACIÓN DE DISCIPLINAS?}

En este trabajo se ha tratado de mostrar que tanto el enfoque generativo como el morfológico son comparativos, en el sentido de que tratan de ofrecer inferencias evolutivas basadas en la comparación entre morfologías.

El enfoque generativo considera que los procesos morfológicos son mecanismos para generar formas (posibles o existentes) y estudiar cómo estas se mantienen y cambian en la evolución. Su estudio experimental ofrecería explicaciones causales y mecanicistas. La asimetría entre los procesos de desarrollo y los patrones evolutivos aparece como un argumento en favor de la autonomía de los procesos, que pueden variar y evolucionar independientemente del patrón al que dar lugar. Aun así, sus defensores consideran que el proyecto de vincular los patrones con los procesos puede mantenerse, aunque de forma mucho más compleja, teniendo en cuenta la enorme variabilidad asociada a los diferentes niveles jerárquicos de la organización viviente, de forma que la variabilidad aparece como patrones acotados de formas morfológicas. La metodología no renuncia al método comparativo aunque consideran que los patrones pueden primeramente detectarse a nivel de taxones superiores (como el locus de la unidad del tipo) de forma que la variabilidad aparezca como «temas» o «variaciones» de un motivo común (ver Wake 1982 y Wake et al. 2011). Se propone avanzar en la comprensión de los mecanismos que deben dar cuenta de la variabilidad morfológica observada asumiendo que deben vincularse los procesos generativos (explanans) con los patrones observados (explanandum). 
El enfoque genealógico se centra en la riqueza y diversidad de los patrones que existen en la naturaleza y la forma en la que estos patrones de desarrollo pueden relacionarse con las explicaciones evolutivas. La independencia evolutiva de patrones y procesos implica que no hay que confiar en una relación causal necesaria entre los estadios ontogenéticos. Los procesos de desarrollo deberían considerarse como caracteres en sí mismos, por lo que se subraya la gran diversidad de las formas en todos los estadios de desarrollo, y que la morfología adulta es un estadio entre muchos. La variabilidad de las formas o las morfologías que aparecen en los procesos filogenéticos u ontogenéticos deben analizarse como los patrones que componen estructuras (espaciales o temporales). Es un método comparativo descriptivo, para el que la exigencia explicativa podría impedir tener en cuenta toda la variabilidad inherente a las formas en la evolución (Scholtz 2008, 2010). Se propone recoger y documentar la riqueza de patrones que existe en la naturaleza de manera que los patrones que aparezcan en el desarrollo puedan relacionarse con los que aparecen en las explicaciones evolutivas sin supuestos previos. Se aboga por una morfología formal separada de toda consideración causal.

Los dos enfoques tienen desacuerdos sobre los méritos del enfoque comparativo y el experimental en morfología, así como sobre la forma de entender el enfoque comparativo y sobre la metodología del análisis y síntesis. Estas diferencias podrían ser particularmente importantes en el contexto del uso de los modelos computacionales de morfologías para el estudio de la evolución, en el que el enfoque genealógico se basaría en un método combinatorio de formas documentadas, mientras que el enfoque generativo se preocuparía por la producción mecanicista de las formas (cercano a la dinámica de formación de patrones).

La discusión de la manera generativa y genealógica de abordar el método comparativo en evo-devo podría tener consecuencias en la forma en que se entienda la integración de las disciplinas biológicas. El enfoque generativo a la manera de Wake o Alberch considera que la integración obliga a las disciplinas que interactúen a cambiar a la luz de los resultados de la otra, convergiendo idealmente en un solo saber que las aúne. El enfoque genealógico que defiende Scholtz podría sostener por su lado que hay motivos importantes para mantener una cierta desintegración de las disciplinas, en el sentido de que la independencia entre las mismas sería ventajosa para garantizar la objetividad de las inferencias evolutivas.

\section{CONCLUSIÓN}

Los desafíos de la dualidad genealógico/generativo en evo-devo sobre si la forma concierne sólo a la descripción o también a la explicación de la diversidad, replantean un problema de gran interés para la filosofía de la ciencia. Un aspecto 
de este tiene que ver con las inferencias evolutivas: ambas perspectivas están en desacuerdo sobre los méritos respectivos del enfoque formal o experimental para estudiar la morfología, o sobre la metodología de análisis exhaustivo de la diversidad orgánica o de síntesis de las formas en base a los descubrimientos sobre la producción dinámica autoorganizativa de las formas. También sostienen posiciones metafísicas dispares sobre la forma y la materia (el enfoque generativo tiende a considerar que la forma orgánica depende de las propiedades dinámicas de la materia), que pueden ser particularmente relevantes en el contexto del uso de modelos computacionales de las morfologías para estudiar la evolución, donde el enfoque genealógico perseguiría necesariamente un enfoque combinatorio, mientras que el generativo sería procesual dinámico. Por último, la manera dispar en la que se concibe el método comparativo desemboca en una concepción integradora o desintegradora de las disciplinas biológicas.

\section{Agradecimientos}

Este texto se desarrolla a partir de lecturas y reflexiones realizadas en colaboración estrecha con Laura Nuño de la Rosa, aunque ella no es responsable de posibles errores o lagunas en el mismo. Agradezco la financiación ofrecida por los proyectos de investigación IT 505-10 del Gobierno Vasco, y FFI2011-25665 del MEC y los fondos FEDER de la UE para la realización del mismo.

\section{REFERENCIAS BIBLIOGRÁFICAS}

ABOUHEIF, E. 2008: «Parallelism as the pattern and process of mesoevolution», Evolution \& Development 10(1): 3-5.

ALBERCH, P. 1985: «Problems with the interpretation of developmental sequences», Systematic Zoology 34(1): 46-58.

,1989: «The logic of monsters: Evidence for internal constraints in development and evolution», Geobois 12: 21-57.

AMUNDSON, R. 2001: «Homology and Homoplasy. A Philosophical Perspective», Encyclopedia of Life Sciences (www.els.net).

BRIGANDT, I. 2002: «Homology and the origin of correspondence», Biology and Philosophy 17: 389-407

ETXEBERRIA, A., NUÑO DE LA ROSA, L. 2009: «Partes y Funciones En El Desarrollo y La Evolución. Hacia Un Darwinismo Sistémico» en H. Dopazo and A. Navarro (Eds.) Evolución y Adaptación: 150 Años Después Del Origen De Las Especies, SESBE: 465-474.

GHISELIN, M.T. 2006: «The failure of morphology to contribute to the modern synthesis», Theory in Biosciences 124: 309-316. 
HALL, B.K. y OLSON, W.M. (Eds.) 2003: Keywords and concepts in Evolutionary Developmental Biology. Cambridge MA: Harvard University Press.

HAMBURGER, V. 1980: «Embryology and the Modern Synthesis in evolutionary theory» en E. Mayr \& W. Provine (eds) The Evolutionary Synthesis: Perspectives on the unification of Biology, New York: Cambridge U.P., pp. 97-112.

JACOB, F. 1977: «Evolution and tinkering», Science 196: 1161-1166.

LALAND, K.N., STERELNY, K., ODLING-SMEE, J., HOPPITT, W., ULLER, T. 2011: «Cause and Effect in Biology Revisited: Is Mayr's Proximate-Ultimate Dichotomy Still Useful?», Science 334: 1512-1516.

MAYR, E. 1961: «Cause and effect in Biology», Science 134: 1501-1506. , 1998: Así es la biología, Barcelona: Debate.

MINELLI, A. 2003: The development of animal form. Ontogeny, morphology and evolution, New York: Cambridge University Press.

„. 2010: «Evolutionary Developmental Biology Does Not Offer Significant Challenge to the Neo-Darwinian Paradigm» en F.J. Ayala y R. Arp (Eds.) Contemporary Debates in Philosophy of Biology, Chichester: Wiley-Blackwell, pp. 213-226.

NEWMAN, S. 2011: «Complexity in organismal evolution» en C. Hooker (ed.) Philosophy of Complex Systems, Elsevier, pp. 335-354.

MÜLLER G. B. y NEWMAN, S. A. (Eds.) 2003): Origination of Organismal Form. Beyond the Gene in Developmental and Evolutionary Biology, Cambridge MA: MIT Press.

NEWMAN, S. A. y COMPER W. D. 1990: "'Generic' physical mechanisms of morphogenesis and pattern formation», Development 110: 1-18.

NUÑO DE LA ROSA, L. 2011: El concepto de forma en la biología contemporanea. Exámen filosófico, Tesis doctoral: Universidad Complutense de Madrid y Université Paris 1 Panthéon-Sorbonne.

NUÑO DE LA ROSA, L y ETXEBERRIA, A. 2012: «Patterns and processes in EvoDevo: descriptions and explanations» en H De Regt, S Okasha, S. Hartmann (Eds.) EPSA 2009, Springer, pp. 263-274.

OWEN R. 2012 [1849]: Discurso sobre la naturaleza de las extremidades (Ed. de S. Balari y G. Lorenzo), Oviedo: KRK Pensamiento.

SCHOLTZ, G. 2008: «On comparisons and causes in evolutionary developmental biology» en G. Minelli and G. Fusco (Eds.) Evolving pathways: Key themes in evolutionary developmental biology, Cambridge: Cambridge University Press, pp. 144-159.

SCHOLTZ, G. 2010: «Deconstructing morphology», Acta Zoologica 91: 44-63.

WAGNER G. 1989: «The biological homology concept», Annual Review of Ecology and Systematics 20: 51-69.

WAGNER, G., CHIU, C-H, LAUBLICHER, M. 2000: «Developmental Evolution as a Mechanistic Science: The inference from Developmental Mechanisms to Evolutionary Processes», American Zoologist 40: 819-831.

WAKE, D. 1982: «Functional and evolutionary morphology», Perspectives in Biology and Medicine 25(4): 603-620. 
, 1991: «Homoplasy: the result of natural selection, or evidence of design limitations?», American Naturalist 138:543-567.

, 2009: «What salamanders have taught us about evolution», Annual Review of Ecology, Evolution, and Systematics, 40, 333-352.

WAKE, D., WAKE M., SPECHT, C.D. 2011: «Homoplasy: From Detecting Pattern to Determining Process and Mechanism of Evolution», Science 331: 1032-1035.

Arantza Etxeberria es Profesora Titular de Lógica y Filosofía de la ciencia en el Departamento de Lógica y Filosofía de la Ciencia de la Universidad del País Vasco, UPV-EHU.

Líneas de investigación:

Filosofía de la biología, filosofía de la medicina, filosofía de la ciencia, sistemas complejos, biología y cognición

\section{Publicaciones recientes:}

SIMON, JR, ETXEBERRIA A., CASADO, A. Eds. 2013: «Special issue: Philosophy of Medicine Roundtable», Theoretical Medicine and Bioethics, (en prensa).

NUÑO DE LA ROSA, L. Y ETXEBERRIA, A. 2012: «»Patterns and processes in EvoDevo: descriptions and explanations, in HW de Regt, S Hartmann \& S Okasha Eds. EPSA Philosophy of Science: Amsterdam 2009, Dordrecht: Springer, pp. 263-274.

Dirección electrónica: arantza.etxeberria@ehu.es 